\title{
1 Selection constrains high rates of satellite DNA mutation in Daphnia pulex
}

2

3 Jullien M. Flynn ${ }^{*}$, Ian Caldas ${ }^{\dagger}$, Melania E. Cristescu ${ }^{\ddagger}$, Andrew G. Clark ${ }^{*}$

4

$5 \quad$ * Department of Molecular Biology and Genetics, Cornell University, Ithaca, USA

$6{ }^{\dagger}$ Department of Biological Statistics and Computational Biology, Cornell University, Ithaca,

7 USA

$8 \quad{ }^{\ddagger}$ Department of Biology, McGill University, Montreal, Canada

9

10

11 Raw data for this manuscript are available at NCBI Sequence Read Archive under BioProjectID:

12 PRJNA341529 BioSamples SAMN05725816 and SAMN05725817. 


\section{RUNNING TITLE: High mutation rates in satellite DNA}

14 KEY WORDS: tandem repeats, mutation accumulation (MA), stabilizing selection, satellite

\section{5 evolution}

16

17 Corresponding author:

18 Jullien Flynn

19 Biotechnology-Rm 223

20526 Campus Road, Ithaca, NY

$21 \quad 14850$

22 jmf422@cornell.edu 


\section{Abstract}

25 A long-standing evolutionary puzzle is that all eukaryotic genomes contain large amounts of

26 tandemly-repeated satellite DNA whose composition varies greatly among even closely related

27 species. To elucidate the evolutionary forces governing satellite dynamics, quantification of the

28 rates and patterns of mutations in satellite DNA copy number and tests of its selective neutrality

29 are necessary. Here we used whole-genome sequences of 28 mutation accumulation (MA) lines

30 of Daphnia pulex in addition to six isolates from a non-MA population originating from the same

31 progenitor to both estimate mutation rates of abundances of satellite sequences and evaluate the

32 selective regime acting upon them. We found that mutation rates of individual satellite sequence

33 "kmers" were both high and highly variable, ranging from additions/deletions of $0.29-105$

34 copies per generation (reflecting changes of $0.12-0.80$ percent per generation). Our results also

35 provide evidence that new kmer sequences are often formed from existing ones. The non-MA

36 population isolates showed a signal of either purifying or stabilizing selection, with $33 \%$ lower

37 variation in kmer abundance on average than the MA lines, although the level of selective

38 constraint was not evenly distributed across all kmers. The changes between many pairs of kmers

39 were correlated, and the pattern of correlations was significantly different between the MA lines

40 and the non-MA population. Our study demonstrates that kmer sequences can experience

41 extremely rapid evolution in abundance, which can lead to high levels of divergence in genome-

42 wide satellite DNA composition between closely related species.

\section{INTRODUCTION}

45 Up to half of the genome of higher eukaryotes is composed of tandem arrays of simple

46 repetitive motifs that can span megabases, called satellite DNA (Platero et al. 1998, Padeken et 
47 al. 2015). Satellite DNA had initially been thought to carry no useful function, and because it

48 posed a replication burden, it became known as "junk DNA" (Orgel and Crick 1980). It also has

49 the potential to be harmful because it can cause deleterious genomic rearrangements facilitated

50 by recombination between similar motifs on different chromosomes (Bzymek and Lovett 2001).

51 Thus, evolutionary biologists have long wondered why repeated sequences accumulate and are

52 maintained at such abundance in the genome. Satellite DNA is typically found in

53 heterochromatic regions where expression is silenced and recombination is low, in $\mathrm{Y}$

54 chromosomes, and in centromeric and telomeric regions (Charlesworth and Charlesworth 2000;

55 Henikoff et al. 2001). One hypothesis is that being situated in heterochromatin, where

56 recombination is suppressed, could both minimize the cost of repeated DNA and reduce the

57 efficacy of selection against it, allowing its accumulation (Charlesworth et al. 1994). Moreover,

58 the observation that, genome-wide, satellite repeats undergo rapid turnover, with nearly complete

59 replacement of these repeat sequences even between closely related species poses challenging

60 questions about the evolutionary forces that shape their evolution (Subirana et al. 2015; Wei et

61 al. 2014). The study of satellite DNA evolution on the timescales of diverged populations and

62 species have revealed interesting patterns, but understanding the forces that generated these

63 patterns requires direct observation on a shorter timescale.

64 Early evolutionary models did not consider selection to be influencing satellite sequence

65 evolution, except perhaps selection on overall genome size (MacGregor and Sessions 1986;

66 Charlesworth et al. 1994; Stephan and Cho 1994). Stephan and Cho (1994) proposed a model

67 where tandemly-repeated sequences could be generated and expand from mutation,

68 recombination, and replication slippage, combined with selection to maintain the genome size.

69 Another model, the "library hypothesis," attempts to explain the differences in satellite 
composition by posing that the common ancestor of related species contained a library of many

71 satellites, which are then differentially amplified within each lineage as they diverge (Fry and

72 Salser 1977). However, the rate at which satellite sequences expand and contract is difficult to

73 quantify. Most models pose evolutionary neutrality of satellite repeats, but we now know that

74 satellite sequences also carry vital cellular functions. Since much of satellite DNA is located in

75 centromeric regions, there is evidence that selection acts on the sequence motifs themselves, as

76 they serve to bind centromere and histone proteins for centromere maintenance and chromosome

77 separation (Henikoff et al. 2001; Malik 2009). These functions have implications for genome

78 stability, and changes in satellite DNA sequences have been shown in some cases to be drivers of

79 speciation by inducing chromosome rearrangements leading to karyotype divergence (Paco et al.

80 2015). Moreover, some satellite sequences are transcribed and may be involved in the regulation

81 of heterochromatin formation (Palomeque and Lorite 2008; Plohl et al. 2008). Even more

82 intriguing is the observation that perturbation of satellite content can alter gene expression

83 genome-wide (Lemos et al. 2008). At present, it is not known whether these examples of

84 functional importance of satellite DNA are the exceptions or the rule and to what extent selection 85 governs the evolution of satellite DNA.

Here, we refer to the units or words of tandemly repeated satellite sequences as "kmers".

87 Several potential and non-mutually exclusive selection regimes could be operating on kmer

88 arrays: negligible selection where satellite DNA content is primarily governed by mutation and

89 drift, stabilizing selection to maintain a particular "optimal" composition, negative selection to

90 purge satellite DNA, and positive selection for rapidly generating new kmer motifs. Studying

91 satellite DNA changes over an evolutionary timescale showed clear differences across

92 geographic subpopulations of a single species and almost complete turnover between species, 
93 confirming that they evolve rapidly (Wei et al. 2014; Subirana et al. 2015). However, quantifying

94 the relative contributions of mutation, genetic drift, and selection is difficult since all these forces

95 are at play in influencing genetic variation in natural populations. Additionally, it has been a

96 challenge to quantify the genome-wide satellite composition since their repetitive nature makes

97 them problematic to sequence and assemble (Hoskins et al. 2007). Early studies describing

98 satellite composition in different species have focused on single satellites or a small family of

99 satellite sequences (Lohe and Brutlag 1987). Obtaining a genome-wide view of the rate of

100 mutation in satellite sequences and how selection shapes their evolution would facilitate the

101 understanding the longstanding puzzles of satellite DNA evolution.

103 bottlenecks every generation and reducing the effective populations size so that both neutral and

104 deleterious mutations can accumulate almost neutrally (Simmons and Crowe 1977). An MA

105 experiment combined with observations from a population with the same genetic background

106 where selection is not removed allows the study of the effects of mutation alone versus the

107 effects of mutation combined with selection (Flynn et al. 2017). Daphnia pulex is an ideal

108 organism in which to study satellite DNA mutation, firstly because MA studies can be conducted

109 effectively under asexual reproduction and single-progeny bottlenecks. Short microsatellite

110 arrays have been studied in D. pulex (Seyfert et al. 2008; Sung et al. 2010), but the sequence

111 motifs and abundances of satellite DNA have not been characterized, although $25 \%$ of the

112 genome is estimated to be heterochromatic (Colbourne et al. 2011). D. pulex has a potentially

113 dynamic genome, with evidence of high rates of deletion and duplication (Keith et al. 2016). The

114 lineage we use reproduces exclusively asexually via apomixis, meaning changes in satellite 
115 content represent spontaneous mutations in the germline without the opportunity for meiotic

116 drive to play a role (Malik 2009; Wei et al. 2017).

117 In this study, we compare the kmer composition of MA lines (without selection) to

118 individuals raised in a competitive non-MA population (with selection) of the identical D. pulex

119 genetic background. We were interested in exploring the mutational dynamics of satellite DNA

120 that can often not be reliably mapped to the reference genome. We quantify the kmer

121 composition using a mapping-independent method to capture all tandem kmers up to $20 \mathrm{bp}$

122 detectable from short-read data, without introducing biases from the reduced representation of

123 repetitive sequences in the reference genome. Our approach allows us to (1) estimate the kmer

124 mutation rates, including expansions, contractions, complete loss of kmers, and generation of

125 new kmers; and (2) evaluate the type of selection (if any) acting on the satellite DNA sequences.

126 Given the observed natural variation in satellite DNA content among populations and species, we

127 expect expansions and contractions to occur at high rates in the MA lines. If satellite DNA is

128 under selective constraint, we expect there to be less variation in kmer abundance in the

129 population evolving under selection than the neutrally-evolving MA lines.

131 MATERIALS AND METHODS

133 Daphnia line setup and DNA sequencing

134 The current study includes a total of 34 genomes: 24 MA line genomes and 6 population isolate

135 genomes that were sequenced in Flynn et al. (2017), and four additional previously unpublished

136 MA genomes. The asexually reproducing MA lines and a non-MA population were initiated

137 from a single progenitor (see Flynn et al. 2017 for a detailed description of the MA experiment). 
138 MA lines were propagated for an average of 82 generations before whole-genome sequencing

139 with single-individual bottlenecks between generations prior to DNA isolation and whole-

140 genome sequencing. In contrast, the non-MA population was maintained without inducing

141 bottlenecks for 46 months in a $15 \mathrm{~L}$ tank, before six individuals were isolated for sequencing.

142 The approximate census population size was estimated to be 100-250. Overlapping generations

143 occurred so the exact number of generations that the population isolates progressed could not be

144 recorded. Thus, we used a life history experiment and estimated the slowest and fastest moving

145 lineages to calculate that the population underwent at least 62 generations (Supplementary

146 Material File S2). Prior to sequencing, Daphnia individuals were subjected to a brief antibiotic

147 treatment and fed with sterile Sephadex beads to reduce contaminants before sequencing (Fields

148 et al. 2015).

Sequencing libraries were prepared with Illumina Nextera procedures in two batches and

150 all genomes were sequenced to approximately the same coverage (10x). To ensure

151 reproducibility between multiple library preparations, we performed technical replication on two

152 MA lines, such that two independent library preparations and Illumina sequencing runs were

153 done for the MA lines C01 and C35 (in a completely separate, third sequencing batch). We

154 analyzed independently all technical replicates to ensure that variation produced by different

155 library preparations is smaller than the variation due to biological expansion/contraction

156 mutations. Libraries were sequenced on an Illumina HiSeq 2000 instrument at Genome Quebec

157 of McGill University with 100 bp paired-end reads.

159 Satellite quantification 
161 To remove redundant sequences, adapters were trimmed and overlapping reads were merged

162 with SeqPrep (https://github.com/jstjohn/SeqPrep). To identify and quantify satellites, the

163 resulting unmapped read files were used as input for the program k-Seek (Wei et al. 2014,

164 https://github.com/weikevinhc/k-seek). The current version of k-Seek detects words or "kmers"

165 of length $1-20 \mathrm{bp}$ that are repeated tandemly to cover at least $50 \mathrm{bp}$ within the same read. The

166 program allows for one single nucleotide mismatch per repetition of each kmer (see Wei et al.

1672014 for details). All offsets and reverse complements of each kmer are compiled and the total of

168 all individual unit copies are summed across all reads. Kmer sequences are presented as the

169 strand/offset that is alphabetically ordered (i.e. A's will be at the start of the sequences if

170 possible). This method of tandem kmer detection and quantification has been found to be

171 reproducible across different library preparations (Wei et al. 2014).

172 To obtain a quantitative comparison between samples we normalized the kmer counts by

173 both individual library sequencing depth and GC content, as PCR-based library preparations are

174 known to show a bias in the GC content of the fragments amplified and sequenced (Benjamini

175 and Speed 2012). This is especially problematic for satellite analysis since, by nature, many of

176 the repeated sequences may have extreme GC contents. First, reads were mapped against the

177 Daphnia pulex reference genome (version 1, Colbourne et al. 2007) using BWA (Li and Durbin

178 2009) v0.7.10 with default settings. Output BAM files spanning the whole genome were given as

179 input to a custom shell script (https://github.com/jmf422/Daphnia-MA-

180 lines/tree/master/GC_correction) to calculate correction factors following Benjamini and Speed

181 (2012). The correction factors produced for high and low GC contents were extreme and

182 variable, which was likely due to low read counts giving unstable estimates as well as mapping

183 biases to the reference genome (Flynn et al. 2017). Thus, to smooth the correction, we used a 
184 Python script to bin values of GC content together, employing wider bins for GC content $<0.25$

185 and > 0.60 (https://github.com/jmf422/Daphnia-MA-lines/tree/master/GC_correction). The

186 correction factor was then applied to kmer counts according to which GC bin their content

187 belonged.

Mutation and interspersion metrics

190 We define mutations in kmer repeats as any change in the number of copies of each specific

191 kmer. The rate of mutation is then the observed change in the number of each kmer per

192 generation. Each kmer may be in tandem arrays found in one or in several locations across the

193 genome, and our method sums the counts of each kmer across all genomic regions. A mutation

194 rate for a given kmer reflects the sum of changes in copy number across these potential multiple

195 loci. To calculate the mutation rates, we used the mean abundance of the kmer in the non-MA

196 population isolates as a proxy for the ancestral abundance using the following equation:

197

$198 u_{i, m}=\frac{m_{i}-\overline{P_{i}}}{G_{m}}$

199

200 Where $u_{i, m}$ is the mutation rate of kmer $i$ in MA line $m, m_{i}$ is the abundance of kmer $i$ in MA line

$201 m, \overline{P_{i}}$ is the mean abundance in the population for kmer $i$, and $G_{m}$ is the number of generations

202 propagated for MA line $m . u$ could be negative (for contractions) or positive (for expansions).

203 We used the same equation to calculate the absolute rate of change of each kmer, except we took

204 the absolute value of the numerator.

As mutation rates could be correlated with copy number, we also calculated the absolute

206 mutation rate normalized by initial copy number using the following equation: 
$u_{i, m}=\frac{\left|m_{i}-\overline{P_{i}}\right|}{G_{m} \times \overline{P_{i}}}$

We used the same equations for calculating the "realized" kmer mutation rates (mutations that made it through selection in the population isolates), and we used the conservative estimate of 62

212 for the number of generations.

We also searched for new kmers generated de novo in the MA experiment. In order to be

214 considered as a new kmer, putative new kmers had to have at least 3 copies in at least one MA

215 line and be completely absent from all other lines. New shared kmers had to have at least 3

216 copies in one MA line and at least 2 copies in a second line. Since k-Seek has a detection limit

217 for kmer arrays that are at least $50 \mathrm{bp}$ long, we checked if the putative new kmers were present in

218 shorter arrays ( $25 \mathrm{bp})$ in the other MA lines to determine if the kmer sequence was generated

219 truly de novo or if it expanded from an already present repeated motif. Similarly, we searched for

220 kmers that had been lost in individual and pairs of MA lines throughout the course of the

221 experiment. Our criteria for identifying lost kmers were that they had to have 0 copies in the

222 affected line or pair of lines, and at least 2 copies in all other lines. We checked if putative lost

223 kmers were completely undetectable or if they were still present in short arrays ( $\sim 25 \mathrm{bp})$, below

224 the detection threshold of k-Seek.

Since we have paired-end reads, we have the potential to detect the level to which kmers

227 interspersion level, we used the metric $I$, as in Wei et al (2014):

$I=n_{i j} / \sqrt{n_{i} n_{j}}$

229 where $n_{i j}$ is the number of read pairs containing kmer $i$ and $\operatorname{kmer} j$, and $n_{i}$ and $n_{j}$ are the number

230 of read pairs containing kmer $i$ and kmer $j$ respectively. 
Data availability

233 Raw sequence files have been deposited in the NCBI Sequence Read Archive BioProject ID:

234 PRJNA341529 BioSamples SAMN05725816 and SAMN05725817.

235 All analyses were performed using RStudio (V 0.99.903), Python V2 and Perl V5 scripts.

236 Html files showing the code and output to all the analyses are available in the Supplementary

237 Material, and all scripts as well as the required processed input files are available at

238 https://github.com/jmf422/Daphnia-MA-lines. File S1 contains descriptions of all supplementary

239 files, as well as the supplementary table and figure legends.

241 RESULTS

\section{Library and GC correction}

244 Over 713 million unmapped reads across all samples were scanned for tandemly-repeated 245 sequences with k-Seek. Of these, over 5.85 million reads $(0.82 \%)$ were found to be composed of

246 kmers of unit length 1-20 bp and encompassed at least $50 \mathrm{bp}$ of the read. Although the second

247 library batch had overall more reads, a similar proportion of the total reads contained kmers in

248 both libraries (Supplementary Figure S1). Unless otherwise noted, the abundance of each kmer is

249 presented in copies per 1x read depth after GC normalization.

250 We found that our qualitative conclusions were robust whether or not we applied a

251 correction factor to normalize the kmer counts based on each kmer's GC content. Our results

252 were also robust across a wide range of parameters used for the GC correction. As library

253 preparations involving PCR have been shown to result in a biased representation of fragments 
254 based on GC content (Benjamini and Speed 2012), we present results after GC correction as

255 described in the Materials and Methods.

256 Since the libraries were prepared in two separate batches, we were concerned that this

257 could confound our results. A Principal Components Analysis (PCA) was able to separate

258 samples based on library batch along PC2, but PC1 appeared to separate samples based on

259 biological mutation patterns (Supplementary Figure S2). There were no consistent differences

260 across all kmers or GC contents of kmers that could be consistently corrected for (Supplementary

261 Material File S4 ). In order to ensure that library batch would not be a confounding factor, we

262 performed a technical replicate of two MA lines and showed that the abundances of each kmer

263 between the library prep batches were highly similar (Supplementary Material File S4).

264 Although there was minor variation between technical replicates in some kmers, their overall

265 mutational patterns were highly similar, shown by their high clustering on PC1 (Figure S2).

266 Additionally, we tested for differences between the mean abundances of the kmers between the

267 MA lines prepared in the first batch and the MA lines prepared in the second batch. The mean

268 abundances of most of the kmers (28 out of 39) in the second-batch MA lines were within the

$26999 \%$ confidence intervals from 1000 subsampling replicates of four first-batch MA lines. 30 of

270 the kmers had mean abundances in the second batch within the range of observed abundances of

271 the first-batch MA lines (Supplementary Material File S4). For these reasons, we did not

272 consider library preparation batch to be a significant confounding factor.

274 Description of satellite DNA content in Daphnia pulex

276 We first sought to describe and quantify the genome-wide short repeat content in Daphnia pulex 
277 and make inferences about kmer origins. There were 162 kmers that had at least 2 copies per $1 \mathrm{x}$

278 coverage in all our D. pulex lines. There were 39 kmers that had an average abundance of at least

279100 copies, and 12 that had an average abundance of at least 1000 copies after normalization

280 (Table S1). We chose to focus most of our analysis on the 39 kmers that had at least 100 copies

281 after normalization (Figure 1). The most abundant kmer, the poly-A repeat, was present at an

282 average of 79,528 copies in each genome. The second most abundant kmer, present at an average

283 of 62,281 copies, was AACCT. This 5-mer is known to be the ancestral telomere repeat in

284 Arthropods (Sahara et al. 2009) and was previously found in Daphnia (Colbourne et al. 2011).

285 Runs of poly-C were also found to be in the "top 39 kmers", having an average of 6379 copies.

286 Two of the four possible 2-mers were included, AG and AC, having an average of 408 and 244

287 copies, respectively. The most abundant kmer sizes were 5-mers (there were six 5-mers of the

288 most abundant 39 kmers), 10-mers (7/39), and 20-mers (7/39). No 15-mers were abundant (>100

289 copies) in the genome, but there were 13 among all 162 kmers. Overall, 20-mers were the most

290 represented with 50 kmers of the 162. Adding up the total short kmer content per genome in base

291 pairs, we found the median to be $1.20 \mathrm{Mb}$ per $1 \mathrm{x}$ coverage, which represents $0.6 \%$ of the

292 estimated $200 \mathrm{Mb}$ genome.

We also compared the satellite composition in D. pulex to that in Drosophila

294 melanogaster, the only other arthropod that has had its genome-wide satellite content

295 characterized to the same level of detail (Wei et al. 2014). This comparison might indicate the

296 extent of satellite diversity across arthropods and functional conservation of some satellites.

297 Comparing the most abundant kmers (at least average 100 copies normalized) between these two

298 species, we found that 10 short kmers (mostly derivations of AG repeats) were present in both

299 species: A, C, AC, AG, AAC, AAG, ACT, AAAAG, AAGAG, and AAGGAG. We found 
several short (3-5 bp) motifs to be highly represented in the most abundant kmers, and among the

301162 kmers (Table 1). Most of these motifs were completely absent or rare within the top $108 D$.

302 melanogaster kmer sequences, except for AG-type motifs (Table 1).

303 In order to understand the origins and dynamics of kmer sequences in Daphnia, we

304 inspected the kmers for sequence similarities. We found that many kmers belonged to families of 305 related sequences separated by a small number of potential mutational steps. Figure 2 shows a 306 network diagram highlighting the potential mutational relationships between 29 of the 39 of the 307 top kmers. The most striking example of a family of related kmers is the 9 kmers of length 10-20 308 bp shown in the top left corner of Figure 2. Ten kmers were not included in the network, either

309 because they were mono-, di-, or tri- nucleotides whose sequence similarities were not

310 meaningful (6 kmers: A, C, AC, AG, AGG, ACT), or because they did not have a clear 311 mutational relationship with any of the other top kmers (4 kmers: AGCCTG, ACAGC, ATCC, 312 AACGGTACGG).

\section{Extremely high rate of mutation in satellite repeats}

315 Because we could not call single nucleotide changes reliably without mapping to the reference 316 genome, we were not able to estimate mutation rates for single base changes in the kmers, and 317 restrict our attention to changes in copy number. To estimate the copy number mutation rates, we 318 used the mean abundance of each kmer in the population isolates as a proxy for the ancestral 319 abundance of the kmer in the MA progenitor. This should be a reasonable estimation assuming 320 that individuals in the population roughly maintained the ancestral kmer content (see below). In

321 fact, all kmers were similar in abundance between the MA lines and the population isolates: all 322 kmer abundances in the population were within 2 standard deviations of the abundance in the 
323 MA lines, and 69\% were within 1 standard deviation. This is consistent with the MA lines both

324 gaining and losing kmer copies. The absolute mutation rates (summing expansions and

325 contractions) per kmer sequence ranged from 0.29 to 105 copies/generation, with a median of

3261.26 copies/generation. This is not including the telomere repeat, which had the highest rate of

327 change at 199 copies/generation. It is likely that the changes affecting the telomere repeats are

328 from a different, non-mutational process (i.e. caused by a mechanism involving telomerase

329 which is not transgenerationally inherited). The suppression of meiosis in these Daphnia lines

330 may have resulted in relaxed selection on telomere maintenance, but we have no direct evidence

331 in support of this.

332 We found that the mutation rates were correlated with the kmer's initial copy number

333 abundance, proxied by the mean abundance of the population isolates, and that the best fit of this

334 relationship is linear $\left(r^{2}=0.88, p<2.2 \times 10^{-16}\right)$. Thus, to compare mutation rates of individual

335 kmers, we normalized the mutation rates by the initial unit copy number to give mutation rates in

336 copies per generation per original copy. Even after this normalization, we still found mutation

337 rates to be exceptionally high - on the order of $10^{-3}$ per copy per generation - compared to

338 previous estimates of other types of mutation such as microsatellite mutations. Normalized

339 mutation rates ranged from $1.23 \times 10^{-3}$ to $7.97 \times 10^{-3}$, with a mean of $2.74 \times 10^{-3}$ copies per

340 generation per original copy. Expansions were more frequent than contractions, with more MA

341 lines experiencing expansions in most kmers, and the overall rate of change was in a positive

342 direction for 124/162 kmers (76\%) (Figure 3a and 3b). Expansion and contraction rates were not

343 correlated with either GC content or kmer size (Supplementary Material Figure S3).

344 A multi-generational MA experiment provides the potential opportunity to observe

345 completely new kmer sequences arising. We searched for kmers that were generated de novo 
346 during the experiment, in single lines and shared across pairs of MA lines. We found five kmers

347 gained in single MA lines and three gained in three independent pairs of MA lines (Table 2).

348 These MA lines did not share single nucleotide mutations within uniquely mapping regions of

349 the genome (Flynn et al. 2017). Five of the eight new kmers seemed to originate from a single

350 base substitution of an existing kmer present in high abundance (Table 2). Two of the other new

351 kmer sequences contained motifs that were abundant in existing kmers. Only 3 out of 8 putative

352 new kmers were present in shorter arrays ( $\sim 25 \mathrm{bp}$, half of k-Seek’s requirement) in the ancestral

353 lineage (Table 2). We also searched for kmers that were lost from individual and pairs of MA

354 lines. There were seven kmers lost uniquely and two lost in a pair of lines (Table 3). Three of the

355 seven lost kmers were lost from MA line C40. C40 is the MA line that was found to have 100

$356 \mathrm{~kb}$ of homozygous deletions of non-repetitive sequence mapped to the reference genome on one

357 chromosome (Flynn et al. 2017), so it is possible that these lost kmers were present exclusively

358 in the deleted regions. Of the lost kmers, 5 out of 7 were still detectable in shorter arrays $(\sim 25$

359 bp) in the affected MA line (Table 3).

361 MA lines versus population with selection

363 Comparing the changes in kmer composition between the MA lines and the population isolates,

364 which experienced selection, reveals the potential influences of selection on satellite DNA. The

365 population provides a valid comparison to the MA lines because it was previously shown to

366 show signs of purifying selection in both single nucleotide mutations (Flynn et al. 2017) and

367 copy number variants (CNVs) (Chain et al. 2017, in prep). The population did not experience a

368 recent bottleneck and in fact the most recent common ancestor of the isolates analyzed here was 
the progenitor of the experiment (Flynn et al. 2017). We also made a conservative estimate of the number of generations the population underwent in order to compare mutation rates

371 (Supplementary File S2). First, we compared the total genome-wide amount of tandem repeats

372 by multiplying the length of the kmer with its number of copies in each genome. The MA lines

373 diverged by a factor of 1.67 in overall kmer abundance, from $1.00 \mathrm{Mb}$ to $1.67 \mathrm{Mb}$ total, with a

374 median of $1.23 \mathrm{Mb}$. The population isolates had both a lower genomic amount of kmers and a

375 narrower range among isolates, ranging from 0.97 to $1.22 \mathrm{Mb}$, with a median of $1.05 \mathrm{Mb}$ (Figure

376 4a). A Levene's test did not detect a significant difference in the variances between these two

377 groups $(\mathrm{p}=0.12)$. However, the mean total abundance (but not the variance) of kmers in the

378 population was below the 5\% confidence interval produced from 1000 replicates of 6 randomly-

379 sampled MA lines (Supplementary Material File S3).

The MA lines underwent expansions across many kmers, with some MA lines

381 experiencing a considerable increase in satellite DNA content in their genome over the course of

382 the MA experiment compared to the non-MA population isolates (Figure 4b). Most notably was

383 MA line C20, which experienced expansions in 34 of the 39 top kmers, with an overall increase

384 in $580.2 \mathrm{~kb}$ in satellite content over 81 generations. On the other hand, population isolates

385 deviated less from an overall balance of expansions and contractions (Figure 4b).

386 To perform a contrast in the variation of individual kmers between the MA lines and the

387 population, we calculated the coefficient of variation $(\mathrm{CV}$, the standard deviation divided by the

388 mean) for each kmer for both the MA lines and the population. We found that the CV was higher

389 in the MA lines than the population for 37 of the top 39 kmers (Figure 4c, $P<2.84 \times 10^{-9}$, sign

390 test). We considered the possibility that the CV of the MA lines was artificially inflated if the

391 mean abundances of some kmers in the second library preparation MA lines were different that 
392 of the first library preparation MA lines. We also considered that the CV may be greater in the

393 MA lines partially because the MA lines may have undergone more generations than the

394 population. In order to ensure these factors were not founding our results, we also calculated the

395 CV of the MA lines comparing only the ones that were prepared in the same library and

396 normalized the $\mathrm{CV}$ of the MA lines by the difference in the generation number between the MA

397 lines and the population. After this conservative calculation, the CV was higher than the

398 population isolates across 25 of the 39 top kmers (Supplementary Material File S3).

399 Next, we compared the realized mutation rate - the mutations that made it through the "filter" of

400 selection in the non-MA population - to the mutation rate in the MA lines. We used the

401 conservative calculation of 62 generations propagated in the population (Supplementary Material

402 File S2). The realized mutation rates in the population were lower than the MA lines for 32 of

403 the top 38 kmers not including the telomere repeat (Figure $4 \mathrm{~d}, P<2.43 \times 10^{-5}$, sign test). Since

404 the sample size of the non-MA population isolates is lower than the sample size of the MA lines

405 (6 versus 28), we performed 1000 subsampling replicates for each kmer by sampling 6 random

406 MA lines and calculating the mean mutation rate. We found that 22 out of $38 \mathrm{kmers}$ had a lower

407 realized mutation rate in the population than the 5\% quantile of the $1000 \mathrm{MA}$ line subsample

408 replicates (Supplementary Material File S3). Moreover, some kmer sequences seemed to be more

409 constrained than others. We roughly quantified constraint by the difference between the mutation

410 rate of the MA lines to the realized mutation rate of the population for each kmer. Five kmers

411 experienced high levels of constraint by this measure, with the realized mutation rate being at

412 least $75 \%$ lower in the population than the MA lines: AGG, C, AGCCTG,

413 AAGCCAGTGCAGC, and AATCTGGAATGGAATGG. All of these highly constrained kmers

414 had statistically significant lower realized mutation rates by the subsampling test above. On the 
415 other hand, 9 kmers seem to have experienced little constraint in their expansions and

416 contractions, with their realized mutation rates in the population being either slightly higher in

417 the population or very close to that of the MA lines (Figure 4d, Supplementary Material File S3).

418 Wei et al. (2014) noted strong patterns of correlation of kmer abundances across different

419 populations of Drosophila melanogaster, suggesting some sort of evolutionary non-

420 independence. These correlation patterns may suggest constraints on the mutational process, or

421 they may be driven by selection. To investigate correlations in the expansions/contractions

422 between kmers, we computed a correlation matrix (using the Pearson correlation) between each

423 kmer for the MA lines and the population. This would indicate correlations between changes in

424 kmer content inherent to mutation as well as correlations caused by similar selective regimes

425 among kmers. We found that some (mainly positive) correlations existed in the MA lines, but the

426 correlations between kmer changes in the population were more common and stronger for both

427 positive and negative correlations (Figure 5a and 5b). A Mantel test indicated that these

428 correlation matrices were significantly different $(P<0.001)$, indicating a role of selection in

429 interactions between satellite sequences.

$430 \quad$ For both the MA lines and the population, strong correlations between kmers that had GC

431 contents between 0.6-0.7 were apparent. Although it is possible that the correlations reflect

432 technical bias, we think this is unlikely because these strong correlations were between the nine

433 kmers closely related in sequence (Figure 2). These nine 10-20 bp kmers have a GC content

434 between 0.6 and 0.7 , and their deviations were in fact strongly positively correlated (Figure 5c).

435 Aggregations of kmers that are related in sequence might easily explain some of the patterns of

436 correlation among kmer sequences that we identified. To follow up on this, we analyzed the

437 paired-end reads to measure the level of interspersion between kmer sequences. All kmers were 
438 highly interspersed with themselves, indicating the that kmer arrays we are studying span at least

439 the length of the sequenced fragments $(\sim 250 \mathrm{bp})$. Several groups of kmers were interspersed with

440 each other (Supplementary Material, File S5). The telomere repeat, unsurprisingly, was not

441 interspersed with any other of the top 39 kmers. The group of nine related kmers mentioned

442 above were the most mutually interspersed as a group. Eight of the nine were interspersed with at

443 least one other in the group, and the more abundant kmers of the group were interspersed with up

444 to six other kmers within the group.

\section{DISCUSSION}

448 To our knowledge this is the first study that assays the mutation rates of genome-wide tandemly

449 repeated satellite DNA using MA lines. The inclusion of data from a population that experienced

450 selection also enables us to begin to detect whether selective forces act on satellite DNA. We

451 found that mutation rates in satellite DNA are high, but they appear to be constrained by

452 selection. We assumed the population isolates maintained the ancestral satellite composition

453 when calculating mutation rates, which was reasonable given that the means of the population

454 and MA lines were similar for most satellites, and the population isolates showed narrow

455 changes in kmer abundance. The high variance in the abundance of kmers across the MA lines

456 resulted in high estimated mutation rates. We are confident that these results are biological and

457 not due to technical biases because we normalized kmer counts by the GC-normalized read

458 coverage (see Materials and Methods), and after normalization, there was no longer a correlation

459 between sequencing depth and kmer abundance (Supplementary Material Figure S4). All MA

460 lines and population isolates contained a similar proportion of reads with kmers to total reads 
461 (Supplementary Material Figure S1). There was also a high level of concordance in the kmer

462 content between technical replicates. Another line of support that these data are reliable for this

463 type of study is that the analysis of copy number variation of mapped reads revealed mutation

464 rate estimates close to those of previous studies (Chain et al. 2017 in prep). Going forward, using

465 PCR-free library preparation methods can reduce batch-based biases in sequencing results (Wei

466 et al. 2017, in prep).

High mutation rates can explain the rapid turnover of satellites

470 The rates of mutation in satellite sequences in our D. pulex MA lines were extremely high,

471 ranging from 0.29 to 105 copy changes per generation for a given kmer sequence. Our mutation

472 rates include the sum of changes across all loci of a particular kmer, thus can include both

473 loss/gain of entire repeat arrays (i.e. from recombination), and changes in the number of repeats

474 within individual loci (i.e. from replication slippage). This makes our study distinct from

475 previous studies of microsatellite mutation rates that have focused on single loci (Seyfert et al.

476 2008). Here, mutation rates estimated on a per-copy basis for satellite repeats we examined were

477 on the order of $10^{-3}$ copies/generation/copy. Seyfert et al. (2008) estimated the per locus mutation

478 rate of D. pulex dinucleotide microsatellites $13-47$ repeats long to be on the order of $10^{-5}$ to $10^{-4}$

479 copies/locus/generation. This indicates not only that many satellite sequence arrays are present as

480 multiple loci and long arrays, but that they mutate at much higher rates than microsatellites

481 typically studied. One contributing factor could be that the short microsatellite arrays are more

482 constrained since they are often found in introns or even coding regions (Ellegren 2004). Past

483 studies of microsatellites have found that rates of mutation are positively correlated with the 
484 number of repeats, but limited data points prevented the determination of a linear relationship

485 (Wierdl et al. 1997; Brinkmann et al. 1998). We had mutation rate estimates for $39 \mathrm{kmer}$

486 sequences ranging in abundance from 100 - 50,000 copies and found a significant positive linear

487 correlation between copy number and mutation rate. This suggests a simple relationship between

488 the number of copy units present and the potential for copy units to be gained or lost, consistent

489 with replication slippage being the dominant mechanism of mutation (Schlötterer 2000). We also

490 found that expansions are more common than contractions, indicating that satellite DNA in

491 Daphnia has the intrinsic tendency to expand, which is in agreement with the consensus finding

492 in microsatellites across taxa (reviewed in Ellegren 2004).

494 satellite DNA sequences between species, and even substantial differences in abundances among

495 diverged populations of the same species. Wei et al. (2014) used k-Seek to characterize the

496 satellite sequences in eight different populations of D. melanogaster, distributed globally. They

497 found that the overall satellite content varied by a factor of 2.5 , accounting for about a $4 \mathrm{Mb}$

498 difference. This initially surprising finding makes sense in light of our results that the MA lines

499 that were only 82 generations diverged differed in their overall satellite content by a factor of

5001.67 , accounting for about $0.67 \mathrm{Mb}$. Even the population isolates, which showed a reduction of

501 variation in satellite content compared to the MA lines, varied in their overall satellite content by

502 a factor of 1.27. This highlights the great potential for expansions and contractions in satellite

503 sequences, which can explain the high levels of differentiation in genome-wide satellite

504 compositions between populations and related species (Wei et al. 2014; Subirana et al. 2015;

505 Wei et al. 2017 in prep). 
Daphnia and Drosophila are both arthropods, although they are estimated to have

507 diverged about 400 million years ago (Rehm et al. 2011). Given the high differentiation observed

508 between closely related species, the paucity of shared sequences and motifs between these taxa is

509 not surprising. It is worth noting, however, that the AGG and AAG motifs as well as a couple

510 AG-rich satellites were conserved between the two species. This conservation could be random

511 or it could indicate a conserved functional role for AG-rich satellites; for example, involvement

512 in binding conserved proteins. The GAGA factor is known to bind to AG-rich satellite sequences

513 in Drosophila, although it has been hypothesized to have evolved in the ancestor of Diptera and

514 Hymenoptera and is not thought to be present in Daphnia (Heger et al. 2013). However, it is

515 possible that other proteins could be playing a role in binding to AG-rich satellites in Daphnia

516 similarly to Drosophila. Another possibility is that AG-rich satellites result in a favourable DNA

517 curvature (Palomeque and Lorite 2008). D. melanogaster populations were found to be enriched

518 in kmers that were multiples of 5 bp long (i.e. 5, 10, 15, 20, -mers) (Wei et al. 2014).

519 Interestingly, we also found that Daphnia were enriched in kmers 5, 10, and 20 bp long.

520 Although there were no 15-mers at high abundance ( $\geq 100$ copies), 15-mers were enriched when

521 considering the dataset of 162 kmers. This pattern, conserved from Drosophila to Daphnia,

522 could indicate conservation in the periodicity of DNA wrapping around histones (Lohe and

523 Brutlag 1987).

Based on the new kmers generated de novo in the MA experiment and the relatedness of

525 existing kmers, we suggest that novel kmers typically result from a point mutation in an already

526 abundant kmer, followed by expansion of the new kmer. This is in agreement with the result

527 from Wei et al. (2017 in prep), who found new kmers originating in different Drosophila species

528 that also differed by only a single nucleotide from a pre-existing kmer. Additionally, satellite 
529 kmers can be generated when they expand from a sequence motif that is already present in only a

530 few copies, as we suggest could have occurred for some of our new kmers (Table 2). Concordant

531 with these hypotheses, we found that two kmers were gained independently in two independent

532 MA lines, demonstrating the high potential for new satellite sequences to be generated from

533 existing ones or from an existing motif, and that parallel independent gains of the same satellite

534 are possible. We detected the newly-generated kmers at low abundance, but after many more

535 generations they would presumably have the potential to expand and achieve higher abundance

536 in the genome. Most of the lost kmers were not completely lost, but contracted to be part of a

537 short enough array to not be detected by k-Seek (Table 3). Some of the satellite losses we found

538 likely resulted from deletions of segments of chromosomes. Flynn et al. (2017) found that the

539 MA line C40 experiences homozygous deletions totalling $\sim 100 \mathrm{Mb}$ on chromosome 11 . Here we

540 found that C40 lost 3 different satellite sequences, so we think it is likely that these satellites

541 were present on the deleted regions of chromosome 11 (although these tandem sequences were

542 not present in tandem in the genome assembly). In fact, C40 experienced a complicated

543 recombination event that resulted in these deletions and complete loss of heterozygosity across

544 the chromosome (Flynn et al. 2017). It is possible that the satellite DNA composition on this

545 chromosome causes it to be a hotspot for structural rearrangements. Nevertheless, if most of our

546 kmer losses resulted from deletions of large segments also containing functionally important

547 regions, these would likely be purged from natural populations and thus would not be detectable

548 in population data. 
552 In contrast to the MA lines, which accumulate mutations with minimal regard to their phenotypic

553 consequences, we found that most kmers experienced constraint in their evolution in the non-MA

554 population. The constraint was disproportionately distributed among kmer sequences, with some

555 being unconstrained in these conditions and others being highly constrained. Both the coefficient

556 of variation and mutation rates were lower for many kmers in the population. The population

557 reproduced asexually and it had a census population size of 100-250, indicating selection would

558 have to be substantially strong in order to produce the signal that we observed. Our results

559 suggest that satellite repeats have the intrinsic tendency to expand in the Daphnia genome, but

560 the magnitude of expansions are reduced under a selection regime. Although this study does not

561 investigate specific functional roles of satellites, we recognize the potential for some satellites to

562 have specific roles (e.g. Fry and Salser 1977; Palomeque and Lorite 2008; Plohl et al. 2008).

563 This may be true for AG-rich satellites, which are known to bind proteins and are conserved in

564 both Drosophila species and D. pulex (Raff et al. 1994). The selection we infer might stem from

565 conservation of a specific role for some satellites, and/or deleterious effects of changing the copy

566 number of satellites.

567 In calculating mutation rates, we assumed that the population maintained the ancestral

568 kmer content. Concordant with this assumption, there was little variation in the total summed

569 abundance of satellite sequences in the population. This would be consistent with the operation

570 of stabilizing selection on overall satellite content (implying that there is an "optimal" level).

571 However, we note that it is possible that negative selection (purging satellite DNA) could have

572 been acting in the population and the lower realized mutation rates in the population was a result

573 of mutation-selection balance (i.e. mutation pressure to increase satellite content, selection to

574 reduce it). Under stabilizing selection, the reduced realized kmer mutation rates in the 
575 population could stem from selection on overall genome size or chromosome organization.

576 Previous studies have shown the importance of satellites for maintaining chromatin organization

577 and that genetic variation in satellite content can affect genome stability and chromosome

578 function (Kim et al. 2009, Aldrup-MacDonald et al. 2016). If the genome has evolved to

579 accommodate satellite arrays, altering the length of an array could alter the chromatin

580 organization and disrupt nearby functional regions, causing deleterious effects. This could be

581 especially important for Daphnia, which has a compact genome with little intergenic space

582 (Colbourne et al. 2011). If selection to remove satellite DNA was the dominant form of selection,

583 this would imply that the satellite arrays were mostly "junk" and the genomes would be in a

584 constant state of purging satellite DNA. Moreover, it seems that the level of selection varies

585 across kmers. We found five kmers to be under very little constraint, shown by the lack of

586 difference between their $\mathrm{CV}$ and mutation rates between the MA lines and population (Figure $4 \mathrm{c}$ 587 and d).

589 Correlations in kmer changes

591 Across the MA lines, we found mainly positive correlations between the kmers in their

592 expansions and contractions. These could have arisen randomly or been driven by physical

593 location/linkage. We found that kmers that were closely related to each other were strongly

594 correlated in their changes in abundance, which was especially evident for the family of 10-20 bp

595 kmers with the GCCAG motif (Figure 2, top left). Concordant with the hypothesis that new

596 satellite sequences come from point mutations in existing ones, we found that these closely

597 related kmers were highly interspersed within each other. Since these positive correlations were 
present in both the MA lines and the population, it is likely to be a consequence of the mutational

process. Segmental duplications and deletions, which are common in Daphnia (Keith et al. 2016), could explain the observed positive correlations. When only the population isolates were

601 considered in the correlations, we identified more correlations of stronger effect, both positive

602 and negative. The positive correlations could arise from the same selective regime acting on

603 pairs of kmers, i.e. if expansions of both kmers of the pair in question are both selected against.

604 On the other hand, the negative correlations may arise if different satellite sequences are in

605 conflict (Wei et al. 2014). Specifically, there could be selection on a chromosome to maintain its

606 organization such that if one kmer happens to expand, another will have to contract. Kmers

607 localized on the same chromosomal region may be the ones negatively correlated with each

608 other, however, we were not able to test this thoroughly with our data because of the un-

609 mappable nature of repeats.

\section{Conclusions}

612 We were able to gain a picture of genome-wide satellite DNA expansions and contractions, and 613 provide evidence that satellite DNA is not always neutrally evolving but can experience strong

614 selection. We also show evidence that new satellite sequences are often generated from existing 615 ones, and there is a complex interaction structure between individual satellites that differs if 616 selection is at play. Future studies using different sequencing technologies, especially with

617 longer reads that can capture longer satellite sequences and span the boundaries of unique and 618 repeated sequences, will provide further insights into satellite DNA evolution. 
621 We would like to thank D. Barbash and F. Chain for useful discussions, and K. Wei for

622 providing scripts for the kmer interspersion analysis. J. Bull provided summary data from the life

623 history experiment that allowed us to calculate the number generations in the non-MA

624 population. We would also like to thank S. Lower for helpful discussions on the analysis of the

625 data and comments that helped improve the quality of the manuscript. The original MA lines and

626 sequencing was supported by NSERC Discovery Grant (341423-2012) to MEC and the kmer

627 analysis was supported by NIH grant R01 GM119125 to AGC and D. Barbash. JMF was

628 supported by a NSERC PGS-D scholarship.

\section{TABLES}

Table 1. Number of kmers that contain certain sequence motifs. The top 39 kmers are characterized by having an average of at least 100 copies per $1 \mathrm{x}$ coverage, and the top $162 \mathrm{kmers}$ have at least 2 copies per $1 x$ coverage in all samples. The D. melanogaster kmers are the 108 kmers that have at least 100 copies normalized (personal communication, K. Wei).

\begin{tabular}{llll}
\hline Motif sequence & In top 39 kmers & In top 162 kmers & $\begin{array}{l}\text { In D. melanogaster } \\
\text { kmers }\end{array}$ \\
\hline AAAA & 4 & 13 & 12 \\
AAC & 7 & 32 & 16 \\
AAG & 11 & 49 & 45 \\
ACGC & 8 & 52 & 0 \\
AGC & 12 & 69 & 4 \\
AGG & 11 & 41 & 14 \\
AGGAG & 3 & 15 & 5 \\
GCCAG & 7 & 48 & 0 \\
TAGG & 4 & 10 & 0 \\
TCCAG & 3 & 14 & 0 \\
\hline
\end{tabular}

632

Table 2. New kmers generated de novo during the MA experiment. Many of them are potentially generated from single nucleotide mutations or rearrangements of existing kmers. It is indicated if the putative new kmer was present in shorter arrays ( $\sim 25 \mathrm{bp})$ in the ancestral lineage.

633

\begin{tabular}{|c|c|c|c|c|c|}
\hline New kmer & Line(s) & $\begin{array}{l}\text { Normalized } \\
\text { abundance }\end{array}$ & $\begin{array}{l}\text { Present in } \\
\text { shorter } \\
\text { arrays }\end{array}$ & $\begin{array}{l}\text { Similar kmer, } \\
\text { or common } \\
\text { motifs (copy }\end{array}$ & $\begin{array}{l}\text { Abundance } \\
\text { of similar } \\
\text { kmer (copy }\end{array}$ \\
\hline
\end{tabular}




\begin{tabular}{|c|c|c|c|c|c|}
\hline & & & & number) & number) \\
\hline AAATG & C18 & 4 & No & $\begin{array}{l}\text { AATGG or } \\
\text { AAAAG }\end{array}$ & $\begin{array}{c}18853 \text { or } \\
471\end{array}$ \\
\hline ACCAG & $\mathrm{C} 02$ & 11 & No & AACAG & 6846 \\
\hline AAACCCTAGTGGGTTGT & $\mathrm{C} 02$ & 14 & No & $\begin{array}{l}\text { AAC, TAGG, } \\
\text { AGG }\end{array}$ & - \\
\hline AAGCCACGC & $\mathrm{C} 02$ & 3 & No & $\begin{array}{l}\text { AAG, AGC, } \\
\text { ACGC }\end{array}$ & - \\
\hline ACAT & C35 & 3 & Yes & - & - \\
\hline AAACT & $\begin{array}{l}\text { C13, } \\
\text { C21 }\end{array}$ & 2,3 & No & AACCT & 62281 \\
\hline AATG & $\begin{array}{l}\text { C12, } \\
\text { C34 }\end{array}$ & 3,5 & Yes & $\begin{array}{l}\text { AAAG or } \\
\text { AAGG }\end{array}$ & $\begin{array}{c}708 \text { or } \\
11\end{array}$ \\
\hline AGAT & $\begin{array}{l}\text { C20, } \\
\text { CC8 }\end{array}$ & 3,2 & Yes & AGAA (AAAG) & 708 \\
\hline
\end{tabular}

Table 3. Kmers that were lost during the course of the MA experiment. It is indicated if the putatively lost kmer is present in short arrays at low frequencies in the MA line in question.

\begin{tabular}{llll}
\hline Lost kmer & Line(s) & $\begin{array}{l}\text { Present in shorter } \\
\text { arrays }\end{array}$ & $\begin{array}{l}\text { Normalized mean } \\
\text { abundance in other } \\
\text { lines (copy number) }\end{array}$ \\
\hline AAGGAATGG & C40 & No & 61.6 \\
AAAAAGAAGGAGATAG & C36 & No & 47.6 \\
AATAG & C25 & Yes & 16.3 \\
ACGCCCGAGC & C40 & Yes & 13.8 \\
AATCT & C20 & Yes & 7.2 \\
ACACCGACCACTACT & C40 & Yes & 5.6 \\
AAAGGCAACAACAGT & C35 & Yes & 4.2 \\
AATGT & C02, C34 & Yes & 12.4 \\
AAAAAAAC & C39, C44 & Yes & 7.5 \\
\hline
\end{tabular}


635

636

637

638

639

640

641

642

643

644

645

646

647

648

649

650

651

652

653

654

655

656

657

\section{FIGURE CAPTIONS}

Figure 1. Abundance of the kmers that contain at least 100 counts after normalization. Kmers are arranged in order of length, from 1-mers to 20-mers.

Figure 2. Network of relatedness of the 29 of the 39 most abundant kmers. Networks show possible mutational steps between related kmers. Kmers are numbered based on their abundance rank. The sizes of the circles also correspond to the kmer abundance.

Figure 3: Kmer mutation rates. (A) Boxplot of mutation rates in the MA lines $(n=28)$. The top 20 kmers are shown, in descending order of abundance. Negative values indicate contractions and positive values indicate expansions. (B) Plot of mean overall mutation rate across all 162 kmers at normalized abundance of at least 2 copies per 1x coverage (in descending order of abundance). The red line indicates an overall mutation rate of 0 .

Figure 4. Reduced variation in kmer content in the population compared to the MA lines. (A) Total absolute amount of tandemly-repeated satellite sequences with length 1-20 bp in the MA lines versus the population, including all 162 kmers with at least 2 copies per $1 \mathrm{x}$ coverage per line. (B) Histogram of the cumulative copy changes across the top 39 kmers in the MA lines and population. (C) The coefficient of variation across the top 39 kmers for the MA lines and the population. Kmers are ordered by abundance. (D) The absolute normalized mutation rate (sum of expansions and contractions), divided by the initial copy number of the kmer. Data from the MA lines are shown in red and the population in blue. 
658 Figure 5. Correlated changes in kmer abundance in (A) the MA lines, and (B) the population.

659 (C) shows the correlations between the family of 9 kmers that are closely related by sequence

660 and GC content, which are situated at the bottom corner of the matrix in B. Matrices were

661 computed from a pairwise Pearson correlation matrix between kmers in the deviation of their

662 abundance from the inferred ancestral abundance. Kmers are ordered by their GC content.

\section{LITERATURE CITED}

Aldrup-MacDonald ME, Kuo ME, Sullivan LL, Chew K, Sullivan BA. 2016. Genomic variation within alpha satellite DNA influences centromere location on human chromosomes with metastable epialleles. Genome Res. 26:1301-1311.

Benjamini Y, Speed TP. 2012. Summarizing and correcting the GC content bias in high-throughput sequencing. Nucleic Acids Res. 40:e72.

Brinkmann B, Klintschar M, Neuhuber F, Hühne J, Rolf B. 1998. Mutation rate in human microsatellites: influence of the structure and length of the tandem repeat. American Journal of Human Genetics. 62:1408-1415.

Bzymek M, Lovett ST. 2001. Instability of repetitive DNA sequences: the role of replication in multiple mechanisms. Proc Natl Acad Sci. 98:8319-8325.

Charlesworth B, Sniegowski P, Stephan W. 1994. The evolutionary dynamics of repetitive DNA in eukaryotes. Nature 371, 215-220.

Charlesworth B, Charlesworth D. 2000. The degeneration of Y chromosomes. Philos Trans R Soc Lond B Biol Sci. 355:1563-1572.

Colbourne JK, Pfrender ME, Gilbert D, Thomas WK, Tucker A, Oakley TH, Tokishita S, Aerts A, Arnold GJ, Basu MK et al. 2011. The ecoresponsive genome of Daphnia pulex. Science. 331:555-561.

Ellegren H. 2004. Microsatellites: simple sequences with complex evolution. Nature Reviews Genetics. $5: 435-445$.

Fields PD, Reisser C, Dukić M, Haag CR, Ebert D. 2015. Genes mirror geography in Daphnia magna. Mol Ecol. 24:4521-4536.

Flynn JM, Chain FJ, Schoen DJ, Cristescu ME. 2017. Spontaneous Mutation Accumulation in Daphnia pulex in Selection-Free vs. Competitive Environments. Mol Biol Evol. 34:160-173.

Fry K, Salser W. 1977. Nucleotide sequences of HS- $\alpha$ satellite DNA from kangaroo rat Dipodomys ordii and characterization of similar sequences in other rodents. Cell. 12:1069-1084. 
Heger P, George R, Wiehe T. 2013. Successive gain of insulator proteins in arthropod evolution. Evolution. 67:2945-2956.

Henikoff S, Ahmad K, Malik HS. 2001. The centromere paradox: stable inheritance with rapidly evolving DNA. Science. 293:1098-1102.

Hoskins RA, Carlson JW, Kennedy C, Acevedo D, Evans-Holm M, Frise E, Wan KH, Park S, MendezLago M, Rossi F et al. 2007. Sequence finishing and mapping of Drosophila melanogaster heterochromatin. Science. 316:1625-1628.

Keith N, Tucker AE, Jackson CE, Sung W, Lucas Lledo JI, Schrider DR, Schaack S, Dudycha JL, Ackerman M, Younge AJ et al. 2016. High mutational rates of large-scale duplication and deletion in Daphnia pulex. Genome Res. 26:60-69.

Kim JH, Ebersole T, Kouprina N, Noskov VN, Ohzeki J, Masumoto H, Mravinac B, Sullivan BA, Pavlicek A, Dovat S et al. 2009. Human gamma-satellite DNA maintains open chromatin structure and protects a transgene from epigenetic silencing. Genome Res. 19:533-544.

Lemos B, Araripe LO, Hartl DL. 2008. Polymorphic Y chromosomes harbor cryptic variation with manifold functional consequences. Science. 319:91-93.

Li H, Durbin R. 2009. Fast and accurate short read alignment with Burrows-Wheeler transform. Bioinformatics. 25:1754-1760.

Lohe AR, Brutlag DL. 1987. Identical satellite DNA sequences in sibling species of Drosophila. J Mol Biol. 194:161-170.

MacGregor HC, Sessions SK. 1986. The biological significance of variation in satellite DNA and heterochromatin in newts of the genus Triturus: an evolutionary perspective. Philos Trans R Soc Lond B Biol Sci. 312:243-259.

Malik HS. 2009. The centromere-drive hypothesis: a simple basis for centromere complexity. In: Anonymous Centromere. Springer. p. 33-52.

Orgel LE, Crick FH. 1980. Selfish DNA: the ultimate parasite. Nature. 284:604-607.

Paço A, Adega F, Meštrović N, Plohl M, Chaves R. 2015. The puzzling character of repetitive DNA in Phodopus genomes (Cricetidae, Rodentia). Chromosome Research. 23:427-440.

Padeken J, Zeller P, Gasser SM. 2015. Repeat DNA in genome organization and stability. Curr Opin Genet Dev. 31:12-19.

Palomeque T, Lorite P. 2008. Satellite DNA in insects: a review. Heredity. 100:564-573.

Platero JS, Csink AK, Quintanilla A, Henikoff S. 1998. Changes in chromosomal localization of heterochromatin-binding proteins during the cell cycle in Drosophila. J Cell Biol. 140:1297-1306. 
Plohl M, Luchetti A, Meštrović N, Mantovani B. 2008. Satellite DNAs between selfishness and functionality: structure, genomics and evolution of tandem repeats in centromeric (hetero) chromatin. Gene. 409:72-82.

Raff JW, Kellum R, Alberts B. 1994. The Drosophila GAGA transcription factor is associated with specific regions of heterochromatin throughout the cell cycle. Embo j. 13:5977-5983.

Rehm P, Borner J, Meusemann K, von Reumont BM, Simon S, Hadrys H, Misof B, Burmester T. 2011. Dating the arthropod tree based on large-scale transcriptome data. Mol Phylogenet Evol. 61:880-887.

Schlötterer C. 2000. Evolutionary dynamics of microsatellite DNA. Chromosoma. 109:365-371.

Simmons MJ, Crow JF. 1977. Mutations affecting fitness in Drosophila populations. Annu Rev Genet. 11:49-78.

Stephan W, Cho S. 1994. Possible role of natural selection in the formation of tandem-repetitive noncoding DNA. Genetics. 136:333-341.

Subirana JA, Albà MM, Messeguer X. 2015. High evolutionary turnover of satellite families in Caenorhabditis. BMC Evolutionary Biology. 15:1.

Sung W, Tucker A, Bergeron RD, Lynch M, Thomas WK. 2010. Simple sequence repeat variation in the Daphnia pulex genome. BMC Genomics. 11:691.

Wei KH, Grenier JK, Barbash DA, Clark AG. 2014. Correlated variation and population differentiation in satellite DNA abundance among lines of Drosophila melanogaster. Proc Natl Acad Sci. 111:1879318798.

Wei KH, Reddy HM, Rathnam C, Lee J, Lin D, Ji S, Mason JM, Clark AG, Barbash DA. 2017. A Pooled Sequencing Approach Identifies a Candidate Meiotic Driver in Drosophila. Genetics. 206:451-465. 
bioRxiv preprint doi: https://doi.org/10.1101/156554; this version posted June 27, 2017. The copyright holder for this preprint (which was not certified by peer review) is the author/funder, who has granted bioRxiv a license to display the preprint in perpetuity. It is made available under aCC-BY 4.0 International license.

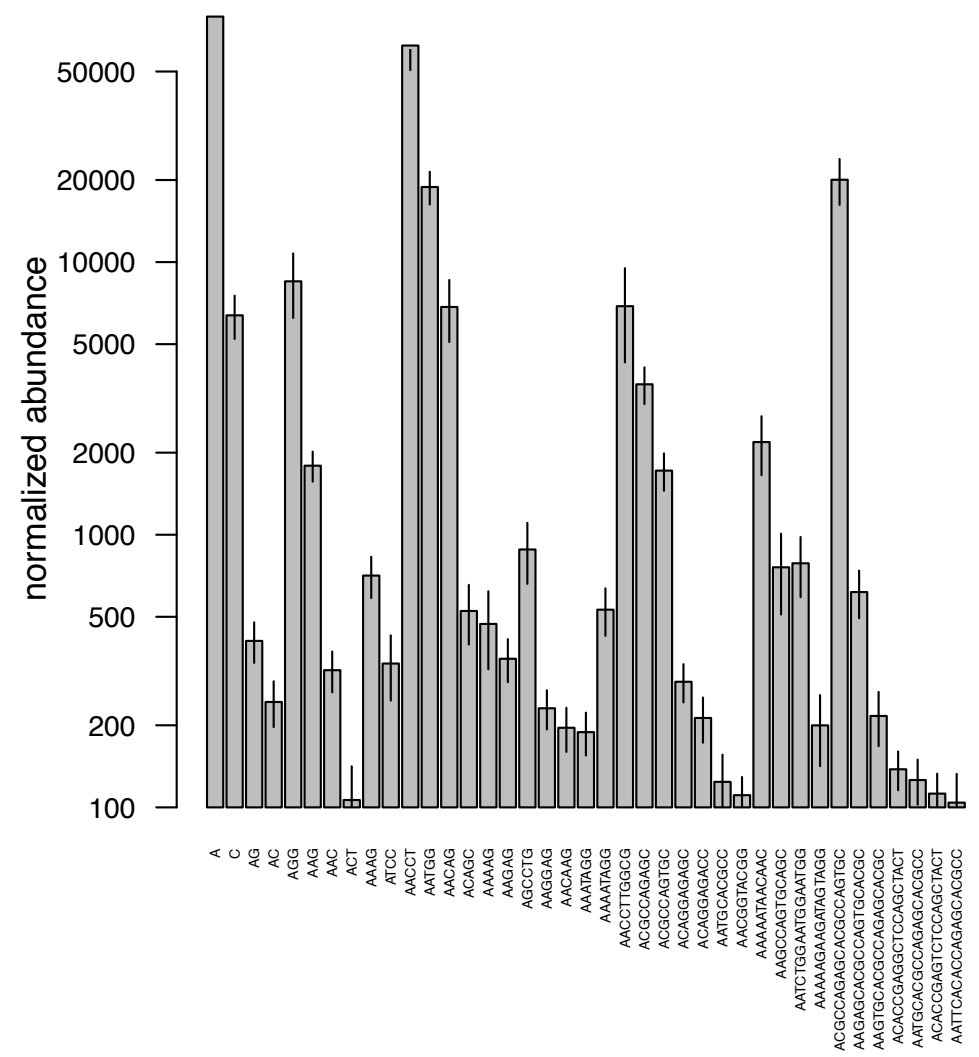

Figure 1.

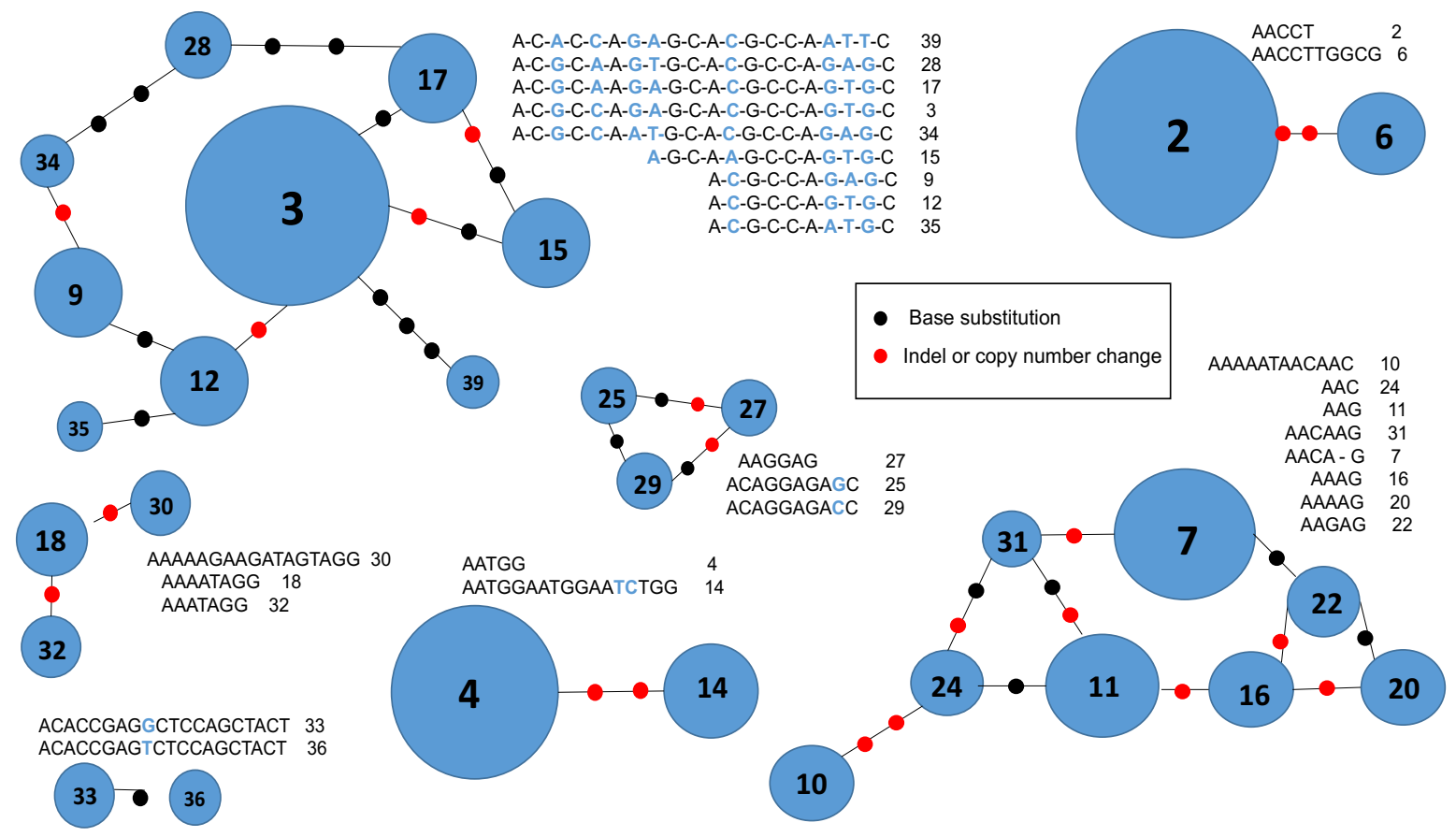

Figure 2. 
$3 A)$

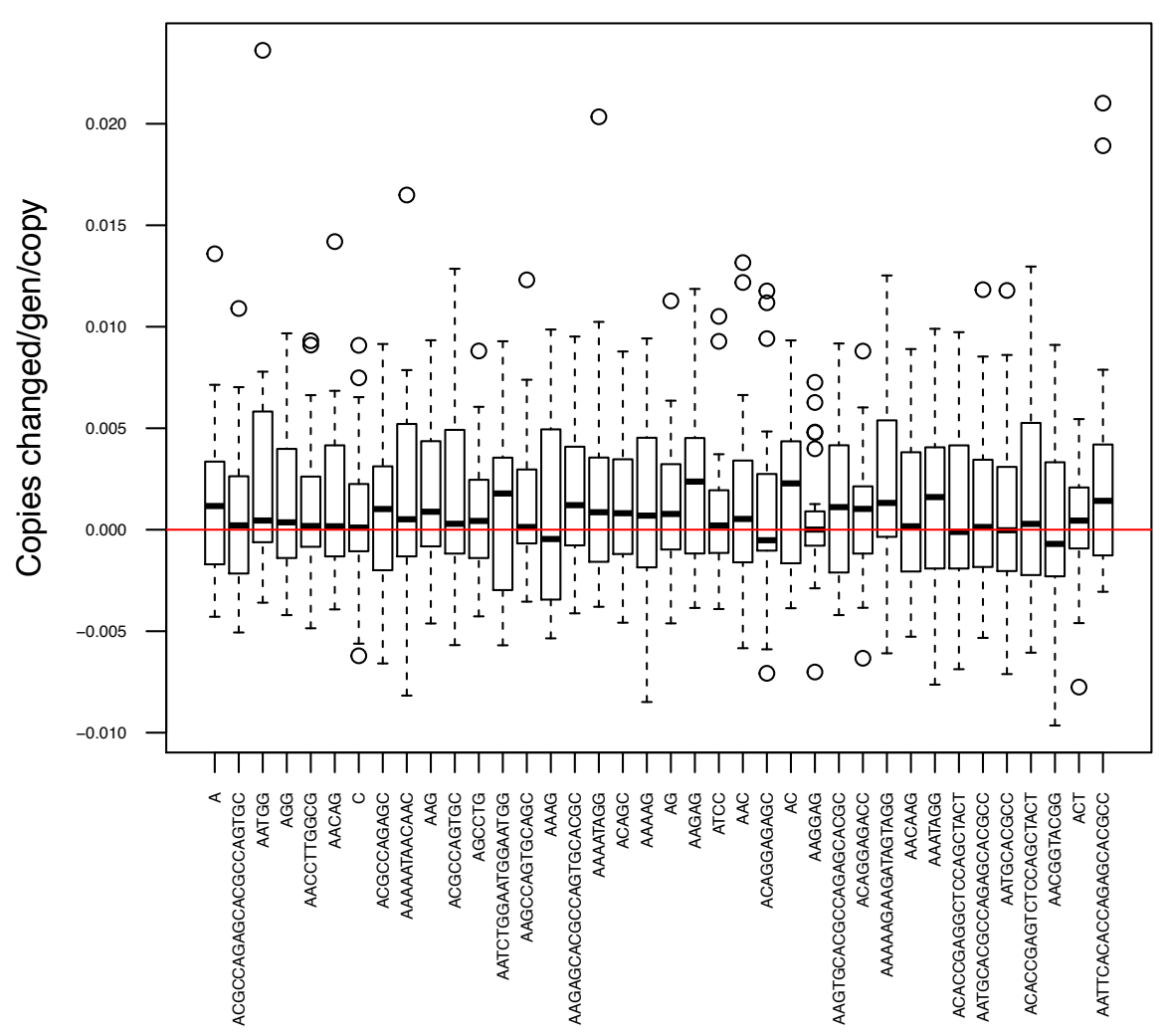

$3 B$

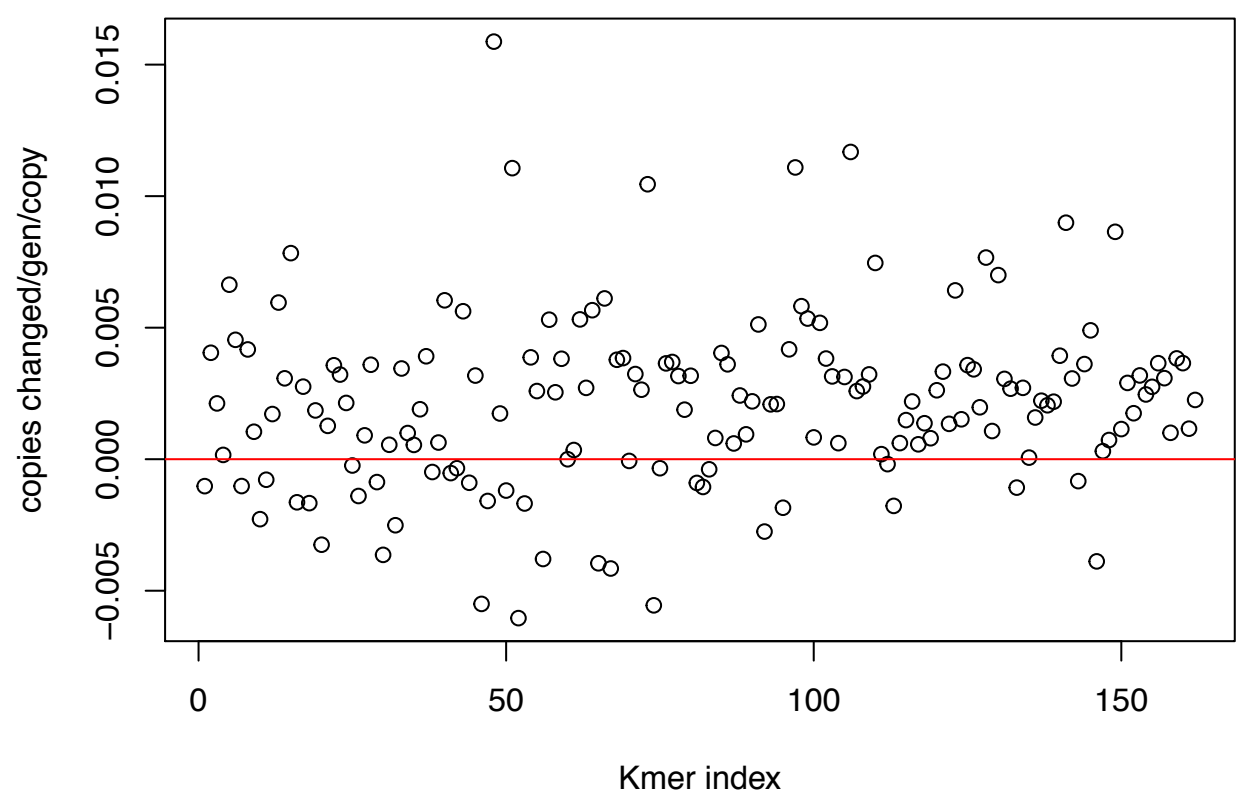

Figure 3. 
bioRxiv preprint doi: $h t t p s: / / d o i . o r g / 10.1101 / 156554$; this version posted June 27,2017 . The copyright holder for this preprint (which was not certified by peer review) is the author/funder, who has granted bioRxiv a license to display the preprint in perpetuity. It is made available under aCC-BY 4.0 International license.

4A)

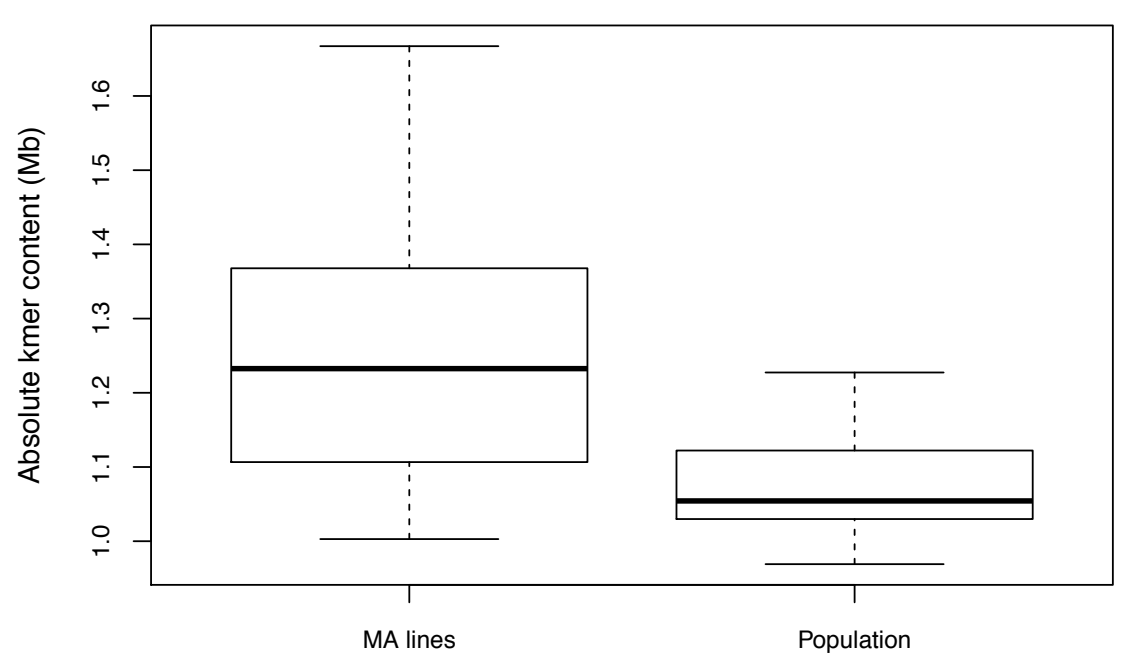

4B)

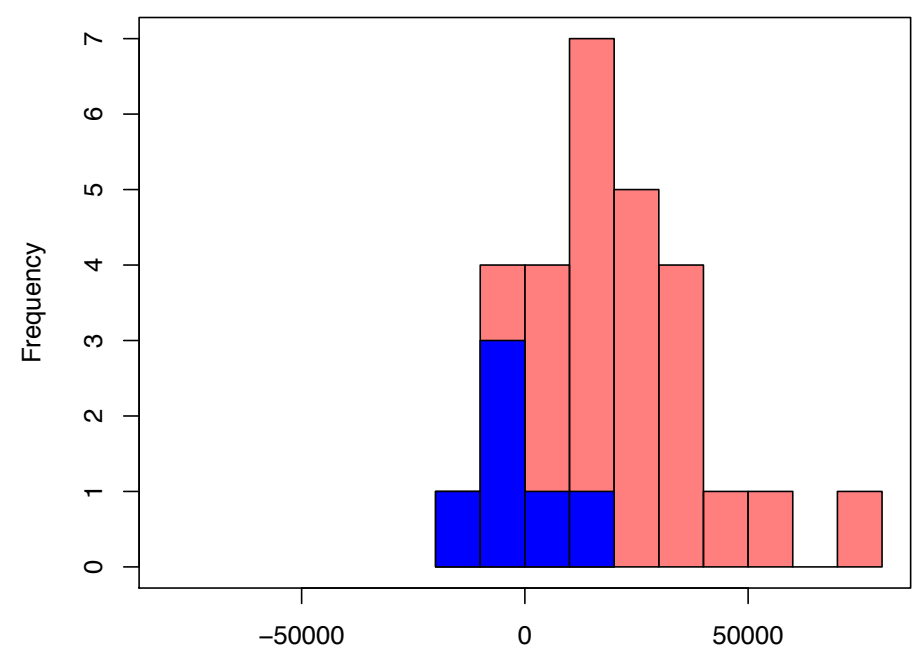

Total copy number change across all kmers 
4C)

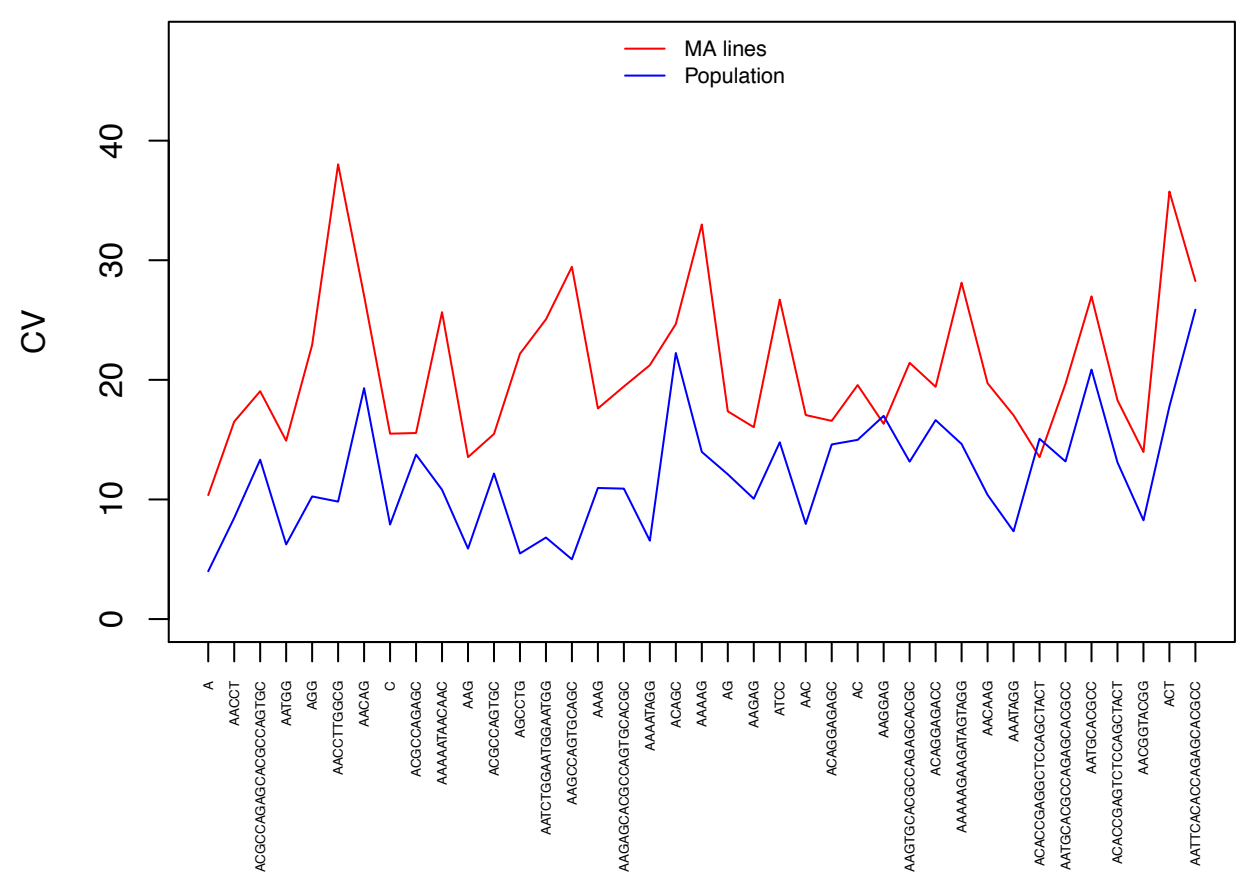

4D)

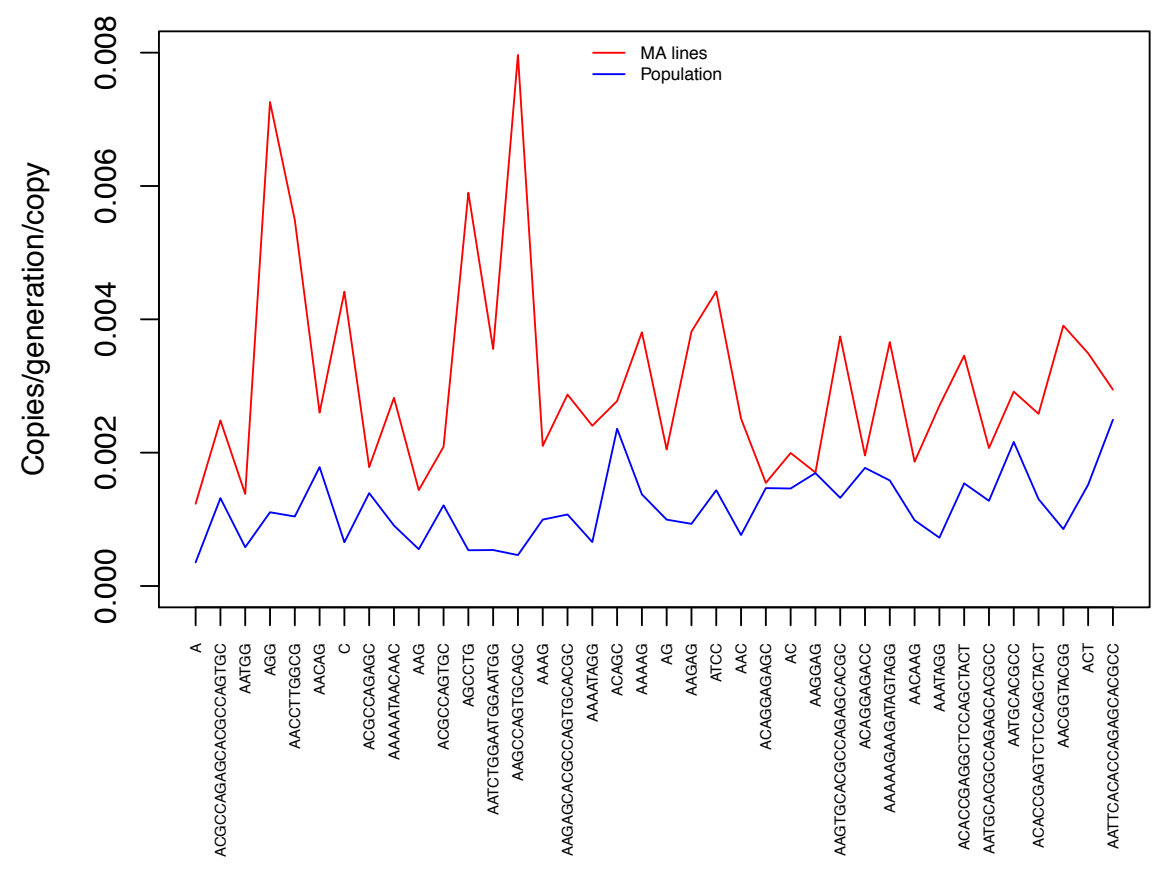

Figure 4. 
bioRxiv preprint doi: https://doi.org/10.1101/156554; this version posted June 27, 2017. The copyright holder for this preprint (which was not certified by peer review) is the author/funder, who has granted bioRxiv a license to display the preprint in perpetuity. It is made available under aCC-BY 4.0 International license.

$5 A)$

5B)
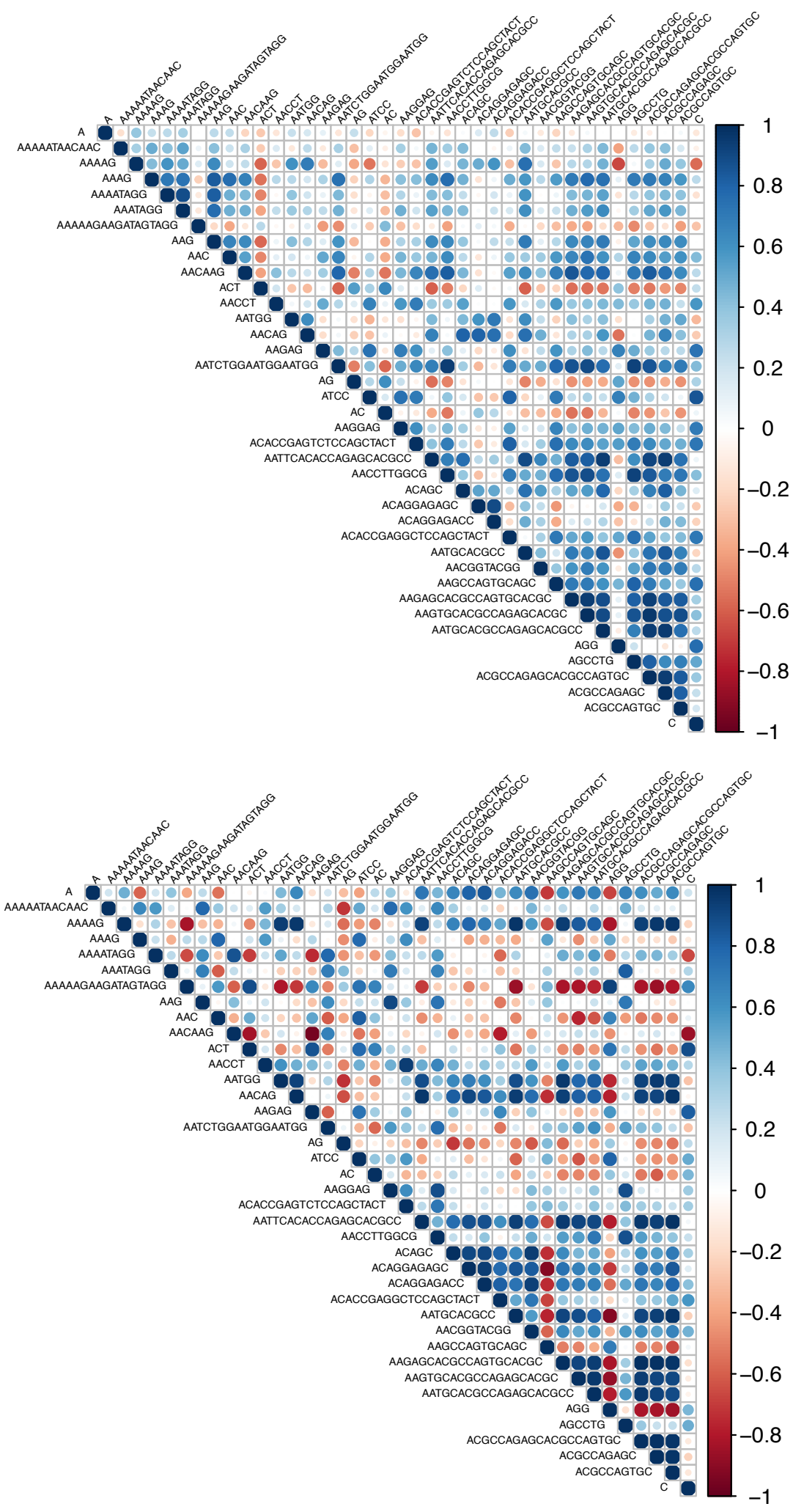
bioRxiv preprint doi: https://doi.org/10.1101/156554; this version posted June 27,2017 . The copyright holder for this preprint (which was not certified by peer review) is the author/funder, who has granted bioRxiv a license to display the preprint in perpetuity. It is made available under aCC-BY 4.0 International license.

5C)

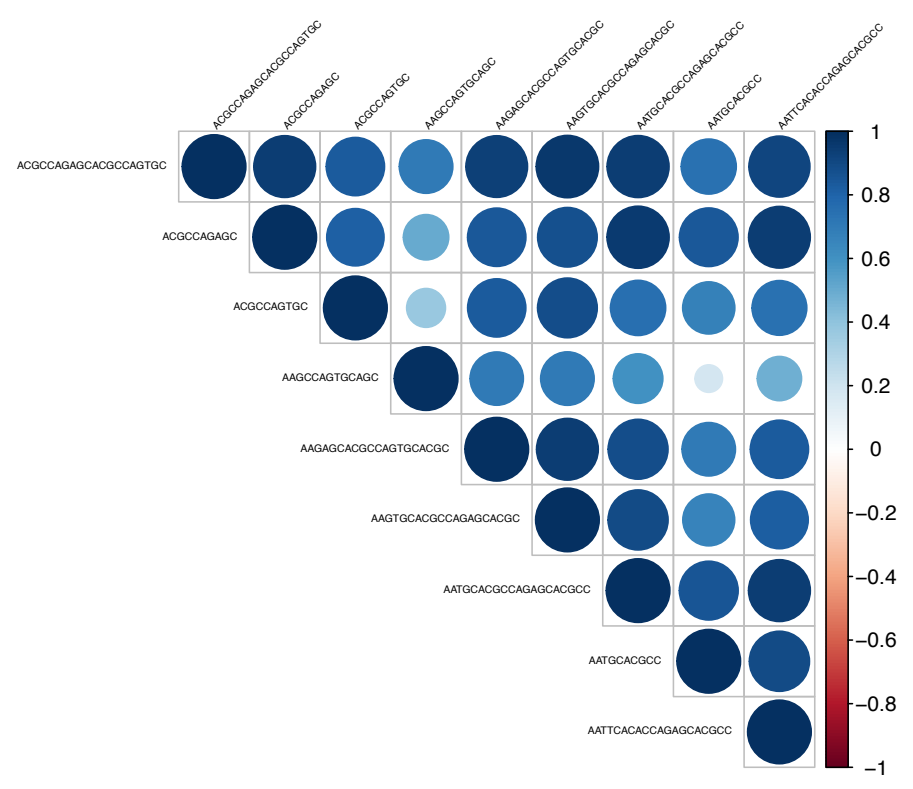

Figure 5. 